\title{
Editorial
}

\section{Joining efforts to destroy a mighty empire: the Delphic oracle reloaded!}

\author{
Carlos Renato Zacharias \\ Editor-in-Chief, International Journal of High Dilution Research (IJHDR)
}

\begin{abstract}
Whether in short or long term, it seems that history always repeats itself! We started in 2011 under the announcement of another collective suicide attempt organized by Merseyside Skeptics Society, with followers in various cities all over the world! The reasoning behind this movement is quite clear: if homeopathic medicines are highly diluted, then it can not be found one even molecule of the supposed active substance inside it. Hence the campaign slogan: Homeopathy: there is nothing in it!
\end{abstract}

This group consists of people who call themselves skeptics, who see homeopathy as a pseudoscience. Theirs arguments are not sustained on mere opinion, but in a classic and enshrined principle by the formally established Science: the limit imposed by the Avogadro constant. This warns us that there is a limit beyond which should we not find more molecules originally present in the starting solution. Thus, the homeopathic remedy should not have an active principle, as it would be consisted by only solvent molecules!

What do this skeptic manifest has to teach us? One might question whether these skeptics have sufficient authority and knowledge to criticize homeopathy or whether their manifestation or organization leads for the convergence or review of some scientific knowledge. But I really believe that such questions would change almost nothing in the knowledge about the phenomenon of high dilutions and homeopathy. So, what lessons should we draw from this episode?

Let us reflect on the basis of their arguments: the Avogadro limit. Based on it, skeptics say, simulating the wisdom of a modern oracle: "respect the Avogadro's limit and we no longer fight against homeopathy"! It is a fact that this restriction has led us to great advances, in Science and Technology as we were able to assign to a material substance, a specific drug effect. And further, describing such a substance in atomic-molecular language!

If homeopathy aims to follow this oracle, it is essential to reduce the homeopathic episteme to classical concepts of the modern science, which would, ultimately, to seek an atomic-molecular origin for the homeopathic action. It would run in consonance to the skeptics claims and the homeopathy would leave the obscurantism of the pseudo-science world, going victorious to the fields of modern science!

And why we do not follow this skeptics' oracle?

The arguments suggested by skeptics obligate us to put the art, science and phenomenology related to the homeopathy, inside an atomic-molecular context regulated by well established rules and classical physicochemical models, widely accepted by the current scientific and technological community. Moreover, this restriction would mean ignoring all the experimental evidences of the biological action of substances that appear to violate the limit of Avogadro.

I agree it is possible to inquire about the quality and accuracy of these experimental evidence, particularly considering we are dealing with an emerging scientific area where the conceptual backgrounds are still to be 
substantiated. But we can not use this same argument to discredit arbitrarily the knowledge produced in the area, nor prevent it to seek and to develop a more consistent formalization.

The evidence for the existence of the phenomenon of high dilutions and support for the homeopathic therapeutics can be roughly classified into two groups: clinical and laboratory evidences.

The clinical evidences are obtained by HPT (Homeopathic Pathogenetic Trials) and meta-analysis of clinical studies that follow classical protocols common to other clinical studies, but adapted to homeopathic episteme. These have shown the validity for using homeopathic medicines and a clear effectiveness in relation to the supposed placebo effect. However it is important to note that such studies do not attempt to describe clinical phenomena from an atomic-molecular basis, but rather whether there is a causal relationship, a clinical action or even a therapeutic potential using highly diluted substances.

On the other hand, there are non-clinical experimental evidences, based on animal experiments, cell cultures, plants, among other models, which have shown us that a HD has a different biological action than those typically expected for an inert solvent, deduced from knowledge supported by the formally established science.

All this evidence seems to demand a new interpretation, different from those conventional!

Perhaps it is important to note that the homeopathic phenomenon was originally realized as a biological event, which was later structured within a conceptual framework. Also, the strongest evidences to validate the existence of such phenomena are of clinical or experimental origin involving biological systems. Physicalchemical experiments, in which a biological sensor is not necessary, have not shown us yet evidences supporting that the atomic-molecular structure of a HD is different from that of the inert solvent.

This should indicate us that we need a new conceptual base to build our reasoning and models! Perhaps no more an atomic-molecular basis restricted to the Avogadro constant, but rather a basis where a relevant part would be the presence of a living being or even a biological sensor able to perceive the information contained in a HD and then to interpret it and finally to express itself in a contextualized response.

However, this does not exclude the requirement of the conceptual and methodological rigor, particularly because we do not know what comes to be such "information" and what are the "main" properties required for a biological sensor. This suggests us thinking in a semiotic model that, although it does not provide us all the answers we want, at least allows us to advance in the researches, free from the restriction imposed by the "Oracle of the Skeptics." But we must keep clear in mind that while we do not give coherent answers to fundamental questions about the homeopathic phenomenon, we will not be "invited" to participate into the current scientific world, despite we are a "natural member" of this community!

The requirement imposed by the skeptics and some other members of the scientific community does not seem to be consistent with the experimental evidences on the homeopathic phenomenon. Do not satisfy the skeptics could seem to indicate that we are definitely relegating the Homeopathy to the world of the pseudo-sciences. What can be done then, by the academic and scientific community that deals with this emerging phenomenon, to win this battle?

I have no doubt that in moments of difficulties the diversity of opinions can be a good strategy to survive! Having different options for action can help us to change positions and see the crisis and difficulties from another point of view, based on new information! But we can not ignore that the focus of discussion is the use of highly diluted solutions!

Surely the researchers engaged in this emerging field are familiar with the "weapons" and rules of this battle, as all were trained by the same academic and scientific structure that currently criticizes us. However, 
despite "weapons" and methods are known, there is a huge numerical and organizational difference in each side of the battle! And perhaps the most importantly, the support of the argument's framework.

What a wonderful battle we could experience where the ultimate goal would be to decide on the feasibility of placing the homeopathy in a state belonging to the formally established science. And the "Oracle of the Skeptics" gives us the hope for victory, because it indicates a strategy for this end: to respect the Avogadro limit and establish a physicochemical basis for homeopathy!

The possibility of this battle reminds me the story of Croesus, the last king of Lydia, that in desiring to combat the spread of the Persian Empire, he consulted the Delphic Oracle that predicted: "if Croesus attacked the Persians, he would destroy a mighty empire"! Supported by the oracle's prediction and strengthened by a military alliance, Croesus launched his campaign against the Persian Empire in 547 BC.

History tells us that Croesus was defeated, fulfilling the prophecy of the oracle: it became clear that the powerful empire Croesus was about to destroy was his own!

It seems that the story wants to repeat! If we join efforts to satisfy the skeptics, the prophecy of the Skeptics' Oracle will be fulfilled: "respect the Avogadro's limit and we no longer fight against the homeopathy"! If we go forward with this fight, we will destroy ourselves, the homeopathy and all science behind the high dilutions, leaving nothing for the skeptics do, unless, quietly to observe our self-destruction!

To respect the limit of the Avogadro's constant is to remain within a biomedical and atomic-molecular framework which does not give us tools to grow up since it is incompatible with the phenomenon we want to describe! To reduce the homeopathy into this framework means destroy the experimental base and evidences of the phenomenon, making impossible to study this issue and progress in this field!

It would then be time to return, to avoid confrontation?

No, because the criticisms are targeting us are not free of bias and reflect the lack of knowledge on the subject by our critics! There are very few critics who invite us to reflect on our own attitudes! And rather than worrying about the critics, we should be concerned with the scientific content we are producing! Homeopathy seems to not have an atomic-molecular basis, as well as the quality of science seems not to be guaranteed by modern scientometric indexes! To answer the call of the skeptics or scientometric indices will not make us grow up, will not make us more organized nor respected!

If we want to get into a true battle, then we should review and reflect on our scientific approach to the phenomenon of the HD and homeopathy. And we don't need to bother in requesting a space in the universe of science, because this decision is not entitled to the skeptics. Further, this place is already booked since a long time! We should only be dedicated to the difficult task of uncovering the phenomenon behind the HD and Homeopathy, in order to fill our space in an honorable manner!

(c)) EY-NC-ND Licensed to GIRI.

How to cite this article: Zacharias CR. Joining efforts to destroy a mighty empire: the Delphic oracle reloaded! [editorial]. Int J High Dilution Res [online]. 2010 [cited YYYY Month dd]; 10(34):1-3. Available from:

http://www.feg.unesp.br/ ojs/index.php/ijhdr/article/view/427/455 . 